\title{
FINITE DIFFERENCE SCHEMES FOR PARTIAL DIFFERENTIAL EQUATIONS WITH WEAK SOLUTIONS AND IRREGULAR COEFFICIENTS
}

\author{
BOŠKO S. JOVANOVIĆ \\ University of Belgrade, Faculty of Mathematics \\ Studentski trg 16, 11000 Belgrade, Serbia and Montenegro
}

\begin{abstract}
A survey of the results concerning the convergence of finite difference schemes for boundary value problems with generalized solutions from the Sobolev space is presented. In particular, difference schemes for some problems with singular coefficients are investigated.
\end{abstract}

2000 Mathematics Subject Classification: 65N15, 65M15.

Keywords: partial differential equation, finite difference scheme, weak solution, rate of convergence.

\section{Introduction}

Finite difference schemes (FDSs) are often used for the approximation of boundary value problems (BVPs) with generalized solutions. In such cases, it is preferable to have the convergence result for the minimal smoothness of input data. This leads to several problems such as: the right hand side of the equation and the solution may be discontinuous functions; the small smoothness of the solution requires the convergence rate estimate in the weak norm; the coefficient of the equation does not belong to the standard Sobolev spaces, etc. In the case of the difference schemes on nonuniform meshes, the order of the local approximation is usually reduced. The accuracy of the method can be increased by using the approximation of the considered differential equation at some non-mesh points and special averaging operators. In the case of problems with singular coefficients, the solution does not belong to the standard Sobolev spaces. Nonstandard conjugation conditions also arise.

In this paper, we give a survey of the techniques for overcoming these problems. Special consideration is given to the derivation of convergence rate estimates consistent with the smoothness of the input data.

The layout of the paper is as follows. In Section 2, the basic concepts of the construction of convergence rate estimates consistent with the smoothness of the input data for FDS approximating problems with weak solutions are presented. Here the Dirichlet boundary value problem for the Poisson equation in a square domain is used as a model example.

Section 3 is devoted to the convergence rate estimates in discrete Sobolev norms $W_{p}^{k}$, for $p \neq 2$.

The technique for establishing convergence rate estimates based on the theory of interpolation of function spaces is presented in Section 4 . 
The difference schemes for elliptic equations with variable coefficients are considered in Section 5. Here it is assumed that the coefficient of the equation belongs to the corresponding spaces of multipliers.

The finite difference scheme for the Helmholtz equation with the youngest coefficient containing the Dirac distribution is investigated in Section 6.

Section 7 is devoted to two-level FDS approximating parabolic initial-boundary value problems.

In Section 8, the heat equation with a concentrated capacity is considered. For the corresponding FDS with averaged right hand side convergence rate estimates consistent with the smoothness of input data in special energy norms are obtained.

Section 9 is devoted to three-level FDS approximating hyperbolic initial-boundary value problems.

In Section 10, the equation of a vibrating string with a concentrated mass is considered.

Section 11 is devoted to the extension of the previous results on nonlinear BVPs. Here for an IBVP for a quasilinear parabolic equation a convergence rate estimate consistent with the smoothness of the input data is presented.

\section{Poisson equation}

As a model problem, we consider the Dirichlet BVP for the Poisson equation in the square $\Omega=(0,1)^{2}$

$$
-\Delta u=f(x), \quad x=\left(x_{1}, x_{2}\right) \in \Omega ; \quad u(x)=0, \quad x \in \Gamma=\partial \Omega
$$

We assume that the solution of BVP (1) is sufficiently smooth, that is, the function $f(x)$ satisfies all the necessary conditions for that.

Let $\bar{\omega}_{h}$ be a uniform mesh in $\bar{\Omega}$ with a step size $h, \omega_{h}=\bar{\omega}_{h} \cap \Omega$ and $\gamma_{h}=\bar{\omega}_{h} \cap \Gamma$. We define the finite differences in the usual way [44]

$$
v_{x_{i}}=\left(v^{+i}-v\right) / h, \quad v_{\bar{x}_{i}}=\left(v-v^{-i}\right) / h
$$

where $v^{ \pm i}(x)=v\left(x \pm h r_{i}\right)$, and $r_{i}$ denotes the unit vector of the $x_{i}$ axis. For the function $v$ defined on the mesh $\vartheta_{h}\left(\omega_{h} \subset \vartheta_{h} \subset \bar{\omega}_{h}\right)$, we define the discrete $L_{2}$-norm

$$
\|v\|_{L_{2}\left(\omega_{h}\right)}^{2}=h^{2} \sum_{x \in \vartheta_{h}} v^{2}(x)
$$

We also introduce the discrete Sobolev norms $\|v\|_{W_{2}^{k}\left(\omega_{h}\right)}(k=1,2, \ldots)$.

We approximate (1) with the standard five-point FDS

$$
-\Delta_{h} v=f, \quad x \in \omega_{h} ; \quad v=0, \quad x \in \gamma_{h} .
$$

The error $z=u-v$ satisfies the conditions

$$
-\Delta_{h} z=\psi, \quad x \in \omega_{h} ; \quad z=0, \quad x \in \gamma_{h},
$$

where

$$
\psi=\Delta u-\Delta_{h} u=\left(\frac{\partial^{2} u}{\partial x_{1}^{2}}-u_{x_{1} \bar{x}_{1}}\right)+\left(\frac{\partial^{2} u}{\partial x_{2}^{2}}-u_{x_{2} \bar{x}_{2}}\right)=\psi_{1}+\psi_{2}
$$


From the inequality (see [46]) $\left\|\Delta_{h} z\right\|_{L_{2}\left(\omega_{h}\right)} \geqslant C_{0}\|z\|_{W_{2}^{2}\left(\omega_{h}\right)}$ immediately follows the a priori estimate

$$
\|z\|_{W_{2}^{2}\left(\omega_{h}\right)} \leqslant C\|\psi\|_{L_{2}\left(\omega_{h}\right)}
$$

By $C$ and $C_{i}$ we denote the positive generic constants independent of $u$ and the mesh stepsize. In different formulas they may take different values. In such a way, to prove the convergence of FDS (2), we must estimate $\psi$. From Taylor's formula $\psi_{i}(x)=\frac{h^{2}}{12} \frac{\partial^{4} u(\tilde{x})}{\partial x_{i}^{4}}$ follows, where $\tilde{x}$ is some midpoint. From here one immediately obtains

$$
\|z\|_{W_{2}^{2}\left(\omega_{h}\right)} \leqslant C h^{2}\|u\|_{C^{4}(\bar{\Omega})} .
$$

A more precise estimate can be obtained using the integral representation of the residual. We have

$$
\begin{aligned}
\psi_{1}(x)=\frac{1}{h^{2}} \int_{x_{1}-h}^{x_{1}+h x_{2}-h} \int_{x_{2}+h} & \left(1-\frac{\left|x_{1}^{\prime}-x_{1}\right|}{h}\right)\left(1-\frac{\left|x_{2}^{\prime}-x_{2}\right|}{h}\right) \\
& \times\left(\int_{x_{1}^{\prime}}^{x_{1}} \int_{0}^{x_{1}^{\prime \prime}} \frac{\partial^{4} u\left(x_{1}^{\prime \prime \prime}, x_{2}^{\prime}\right)}{\partial x_{1}^{4}} d x_{1}^{\prime \prime \prime} d x_{1}^{\prime \prime}+\int_{x_{1}^{\prime}}^{x_{1}} \int_{x_{2}^{\prime}}^{x_{2}} \frac{\partial^{4} u\left(x_{1}^{\prime \prime}, x_{2}^{\prime \prime}\right)}{\partial x_{1}^{3} \partial x_{2}} d x_{2}^{\prime \prime} d x_{1}^{\prime \prime}\right) d x_{2}^{\prime} d x_{1}^{\prime}
\end{aligned}
$$

and an analogous formula for $\psi_{2}$. Therefrom $|\psi(x)| \leqslant C h\|u\|_{W_{2}^{4}(e)}$ where $e=\left(x_{1}-\right.$ $\left.h, x_{1}+h\right) \times\left(x_{2}-h, x_{2}+h\right)$ follows. Summing over the mesh $\omega_{h}$, one obtains $\|\psi\|_{L_{2}\left(\omega_{h}\right)} \leqslant$ $C h^{2}\|u\|_{W_{2}^{4}(\Omega)}$, wherefrom

$$
\|z\|_{W_{2}^{2}\left(\omega_{h}\right)} \leqslant C h^{2}\|u\|_{W_{2}^{4}(\Omega)}
$$

follows.

Estimate (5) can also be obtained by using the Bramble-Hilbert lemma (see $[3,7]$ ). Moreover, as the value of $\psi$ in the node $x \in \omega$ is a bounded linear functional of $u \in W_{2}^{s}(e)$, for $s>3$, which vanishes on polynomials of third degree, applying the generalized BrambleHilbert lemma [7], we obtain

$$
|\psi(x)| \leqslant C h^{s-3}|u|_{W_{2}^{s}(e)}, \quad 3<s \leqslant 4 .
$$

From here, after summation over the mesh $\omega_{h}$ we obtain a more general result

$$
\|z\|_{W_{2}^{2}\left(\omega_{h}\right)} \leqslant C h^{s-2}|u|_{W_{2}^{s}(\Omega)}
$$

for $3<s \leqslant 4$.

For $s \leqslant 3$, the right hand side of (1) and (2) may be discontinuous functions, and consequently, FDS (2) is not well defined. To obtain a well-defined FDS, we average $f(x)$ using the Steklov averaging operators

$$
T_{i} f(x)=T_{i}^{-} f\left(x+0.5 h r_{i}\right)=T_{i}^{+} f\left(x-0.5 h r_{i}\right)=\int_{-1 / 2}^{1 / 2} f\left(x+h y r_{i}\right) d y
$$

These operators commute and satisfy the following relations:

$$
T_{i}^{+} T_{i}^{-}=T_{i}^{2}, \quad T_{i}^{-} \frac{\partial f}{\partial x_{i}}=f_{\bar{x}_{i}}, \quad T_{i}^{+} \frac{\partial f}{\partial x_{i}}=f_{x_{i}}, \quad T_{i}^{2} \frac{\partial^{2} f}{\partial x_{i}^{2}}=f_{x_{i} \bar{x}_{i}} .
$$


For $s<2$, the convergence of FDS (2) does not follow from (6). Consequently, weaker norms must be used to prove the convergence. The following assertion is valid (see $[9,46])$.

Lemma 1. If in (3) $\psi=\eta_{1, \bar{x}_{1}}+\eta_{2, \bar{x}_{2}}$, then

$$
\|z\|_{W_{2}^{1}\left(\omega_{h}\right)} \leqslant C\left(\left\|\eta_{1}\right\|_{L_{2}\left(\omega_{h}\right)}+\left\|\eta_{2}\right\|_{L_{2}\left(\omega_{h}\right)}\right) .
$$

If $\psi=\zeta_{1, x_{1} \bar{x}_{1}}+\zeta_{2, x_{2} \bar{x}_{2}}$ and $\zeta_{i}=0$ for $x_{i}=0$ then

$$
\|z\|_{L_{2}\left(\omega_{h}\right)} \leqslant C\left(\left\|\zeta_{1}\right\|_{L_{2}\left(\omega_{h}\right)}+\left\|\zeta_{2}\right\|_{L_{2}\left(\omega_{h}\right)}\right)
$$

Let us consider FDS with an averaged right hand side [9]

$$
-\Delta_{h} v=T_{1}^{2} T_{2}^{2} f, \quad x \in \omega_{h} ; \quad v=0, \quad x \in \gamma_{h} .
$$

The error $z=u-v$ satisfies conditions (3), where $\psi=\psi_{1}+\psi_{2}, \quad \psi_{i}=\zeta_{i, x_{i} \bar{x}_{i}}, \quad \zeta_{i}=$ $T_{3-i}^{2} u-u, \quad i=1,2$. By Lemma 1 one obtains a priori estimates (4), (8) and

$$
\|z\|_{W_{2}^{1}\left(\omega_{h}\right)} \leqslant C\left(\left\|\zeta_{1, x_{1}}\right\|_{L_{2}\left(\omega_{h}\right)}+\left\|\zeta_{2, x_{2}}\right\|_{L_{2}\left(\omega_{h}\right)}\right) .
$$

Using the Dupont-Scott lemma, as in the previous case, one obtains the following convergence rate estimates: $(6)$ for $2 \leqslant s \leqslant 4$,

$$
\|z\|_{W_{2}^{1}\left(\omega_{h}\right)} \leqslant C h^{s-1}\|u\|_{W_{2}^{s}(\Omega)}, \quad 1<s \leqslant 3,
$$

and

$$
\|z\|_{L_{2}\left(\omega_{h}\right)} \leqslant C h^{s}\|u\|_{W_{2}^{s}(\Omega)}, \quad 1<s \leqslant 2 .
$$

In the case that $0<s \leqslant 1$, the solution of (1) may be a non-continuous function. Let us consider the FDS

$$
-\Delta_{h} u=T_{1}^{3} T_{2}^{3} f, \quad x \in \omega_{h} ; \quad v=0, \quad x \in \gamma_{h}
$$

and define the error in the following manner: $z=T_{1} T_{2} u-v$. As in the previous cases, one obtains estimate (11) for $0<s \leqslant 2[9]$.

Analogous results hold for the FDSs on non-uniform meshes. For example, in [21] for a family of nine-points difference schemes approximating BVP (1) on an arbitrary non-uniform rectangular mesh $\hat{\omega}_{h}$ convergence rate estimates

$$
\|z\|_{W_{2}^{1}\left(\hat{\omega}_{h}\right)} \leqslant C h_{\max }^{2}\|u\|_{W_{2}^{3}(\Omega)}, \quad\|z\|_{L_{2}\left(\hat{\omega}_{h}\right)} \leqslant C h_{\max }^{2}\|u\|_{W_{2}^{2}(\Omega)}
$$

are obtained.

Convergence rate estimates of the form

$$
\|z\|_{W_{2}^{k}\left(\omega_{h}\right)} \leqslant C h^{s-k}\|u\|_{W_{2}^{s}(\Omega)}, \quad s \geqslant k
$$

are called consistent with the smoothness of the solution of BVP (1) (see [37]). Such estimates for the Poisson equation were obtained in $[9,34,53]$, etc. The procedure for the determination of constants in the inequalities following from the Bramble-Hilbert lemma, using the mappings of elementary rectangles onto the standard ones, was suggested in [34]. 
In $[34,36,40]$, the convergence of FDS for the Poisson equation in cylindrical, polar and spherical coordinates was examined and estimates of the type of (12) in the corresponding weighted Sobolev spaces were obtained. FDSs with an enhanced accuracy for equations with constant coefficients were investigated in $[27,52]$. FDSs for the biharmonic equation were considered in $[8,35]$ and for the equations of the elasticity theory - in [33]. An FDS for the elliptic equation with a nonlocal (integral) boundary condition was considered in [2]. Extensive bibliographies on this subject can be found in $[14,46]$.

Note that estimates of the type of (12) are very close to the standard convergence rate estimates for the finite element method [5].

\section{Estimates in $\mathbf{W}_{p}^{k}$ norms}

Let us consider again BVP (1) assuming that its solution belongs to the Sobolev space $W_{p}^{s}(\Omega)$, $1<p<\infty$. As in the previous case, we approximate (1) with the FDS (9). The error $z=$ $u-v$ satisfies conditions (3), where: $\psi=\psi_{1}+\psi_{2}, \quad \psi_{i}=\zeta_{i, x_{i} \bar{x}_{i}}, \quad \zeta_{i}=T_{3-i}^{2} u-u, \quad i=1,2$.

For the function $v$ defined on the mesh $\vartheta_{h}\left(\omega_{h} \subset \vartheta_{h} \subset \bar{\omega}_{h}\right)$, we define the discrete $L_{p^{-}}$ norm with $\|v\|_{L_{p}\left(\omega_{h}\right)}=\left(h^{2} \sum_{x \in \vartheta_{h}}|v(x)|^{p}\right)^{1 / p}, 1 \leqslant p<+\infty$. Analogously we define the discrete Sobolev norms $\|v\|_{W_{p}^{k}\left(\omega_{h}\right)}(k=1,2, \ldots)$.

The following analog of Lemma 1 can be proved using the theory of discrete Fourier multipliers [43].

Lemma 2. FDS (3) satisfies the a priori estimate

$$
\|z\|_{W_{p}^{2}\left(\omega_{h}\right)} \leqslant C\|\psi\|_{L_{p}\left(\omega_{h}\right)} .
$$

If $\psi=\eta_{1, \bar{x}_{1}}+\eta_{2, \bar{x}_{2}}$, then

$$
\|z\|_{W_{p}^{1}\left(\omega_{h}\right)} \leqslant C\left(\left\|\eta_{1}\right\|_{L_{p}\left(\omega_{h}\right)}+\left\|\eta_{2}\right\|_{L_{p}\left(\omega_{h}\right)}\right) .
$$

If $\psi=\zeta_{1, x_{1} \bar{x}_{1}}+\zeta_{2, x_{2} \bar{x}_{2}}$ and $\zeta_{i}=0$ for $x_{i}=0$ then

$$
\|z\|_{L_{p}\left(\omega_{h}\right)} \leqslant C\left(\left\|\zeta_{1}\right\|_{L_{p}\left(\omega_{h}\right)}+\left\|\zeta_{2}\right\|_{L_{p}\left(\omega_{h}\right)}\right) .
$$

Estimating $\zeta_{i, x_{i} \bar{x}_{i}}, \zeta_{i, x_{i}}$ and $\zeta_{i}$ by the Dupont-Scott lemma, one obtains the following convergence rate estimates $[6,49]$ :

$$
\begin{aligned}
\|z\|_{W_{p}^{2}\left(\omega_{h}\right)} \leqslant C h^{s-2}\|u\|_{W_{p}^{s}(\Omega)}, & 2<s \leqslant 4, \\
\|z\|_{W_{p}^{1}\left(\omega_{h}\right)} \leqslant C h^{s-1}\|u\|_{W_{p}^{s}(\Omega)}, & \max \{1,2 / p\}<s \leqslant 3, \\
\|z\|_{L_{p}\left(\omega_{h}\right)} \leqslant C h^{s}\|u\|_{W_{p}^{s}(\Omega)}, & 2 / p<s \leqslant 2 .
\end{aligned}
$$

In the case where $0<s \leqslant 2 / p$, the solution of BVP (1) may be a non-continuous function. In this case, we may define the error as $z=T_{1} T_{2} u-v$ and consider the FDS with a stronger averaged right hand side.

\section{Technique based on the interpolation of Hilbert spaces}

As we have seen, for integer values of the smoothness parameter $s$ convergence rate estimates can be constructed "elementarily", without the Bramble-Hilbert lemma. Using such 
estimates and the interpolation theory of Hilbert spaces [38], one easily obtains the corresponding estimates for non-integer $s$.

Let $X$ and $Y$ be two Hilbert spaces and let $X$ be continuously imbedded in $Y$. Let $0<\theta<1$ and let $[X, Y]_{\theta}$ denote the intermediate space obtained by interpolation [38]. Then $X \subset[X, Y]_{\theta} \subset Y$ and for every $u \in X$ the inequality

$$
\|u\|_{[X, Y]_{\theta}} \leqslant C_{\theta}\|u\|_{X}^{1-\theta}\|u\|_{Y}^{\theta}
$$

holds.

Let $W_{2}^{s}(\Omega)$ be standard Sobolev spaces in $\Omega$. Let us also introduce the spaces $W_{2}^{s}\left((0, T) ; W_{2}^{r}(\Omega)\right)$ and anisotropic Sobolev spaces in $Q=\Omega \times(0, T): \quad W_{2}^{s, r}(Q)=$ $W_{2}^{0}\left((0, T) ; W_{2}^{s}(\Omega)\right) \cap W_{2}^{r}\left((0, T) ; W_{2}^{0}(\Omega)\right)$.

Lemma 3. Let $s_{1}, s_{2}, r_{1}, r_{2} \geqslant 0$ and $0<\theta<1$. Then

$$
\left[W_{2}^{s_{1}}(\Omega), W_{2}^{s_{2}}(\Omega)\right]_{\theta}=W_{2}^{(1-\theta) s_{1}+\theta s_{2}}(\Omega)
$$

and

$$
\left[W_{2}^{s_{1}}\left((0, T) ; W_{2}^{r_{1}}(\Omega)\right), W_{2}^{s_{2}}\left((0, T) ; W_{2}^{r_{2}}(\Omega)\right)\right]_{\theta}=W_{2}^{(1-\theta) s_{1}+\theta s_{2}}\left((0, T) ; W_{2}^{(1-\theta) r_{1}+\theta r_{2}}(\Omega)\right) .
$$

Lemma 4 [38]. Let $A$ be a bounded linear operator from $X_{i}$ into $Y_{i}(i=0,1)$. Then $A$ is also a bounded linear operator from $\left[X_{0}, X_{1}\right]_{\theta}$ into $\left[Y_{0}, Y_{1}\right]_{\theta}$ and the following relation holds:

$$
\|A\|_{\left[X_{0}, X_{1}\right]_{\theta} \rightarrow\left[Y_{0}, Y_{1}\right]_{\theta}} \leqslant C_{\theta}\|A\|_{X_{0} \rightarrow X_{1}}^{1-\theta}\|A\|_{Y_{0} \rightarrow Y_{1}}^{\theta} .
$$

Let us consider again FDS (9). As in the Section 2, one easily shows that

$$
\|z\|_{W_{2}^{2}\left(\omega_{h}\right)} \leqslant C h^{2}\|u\|_{W_{2}^{4}(\Omega)} \quad \text { and } \quad\|z\|_{W_{2}^{2}\left(\omega_{h}\right)} \leqslant C\|u\|_{W_{2}^{2}(\Omega)},
$$

wherefrom, using Lemma 4, one immediately obtains estimate (6) for $2 \leqslant s \leqslant 4$. In an analogous manner one obtains convergence rate estimates in other discrete norms (see $[15,16])$.

The interpolation technique can be used for deriving convergence rate estimates for the FDS approximating various types of boundary value problems, in different function space norms. For example, interesting convergence rate estimates for finite-element and finitedifference methods for solving mathematical physics problems with non-smooth data were derived in $[56,57]$. Convergence rate estimates for the general elliptic convection-diffusion equation with generalized solutions from Nikolskii spaces were obtained in [58].

\section{Equations with variable coefficients}

Let us now consider the elliptic equation with variable coefficients

$$
\mathcal{L} u \equiv-\sum_{i, j=1}^{2} \frac{\partial}{\partial x_{i}}\left(a_{i j} \frac{\partial u}{\partial x_{j}}\right)=f(x), \quad x \in \Omega
$$

with a homogeneous Dirichlet boundary condition. We assume that $u \in W_{2}^{s}(\Omega)$ and $f \in$ $W_{2}^{s-2}(\Omega)$. 
Let $V$ and $W$ be two function spaces in the same domain. The space of multipliers $M(V, W)$ is defined by: $M(V, W)=\{a(x): a(x) v(x) \in W, \forall v(x) \in V\}, M(V)=$ $M(V, V)$ (see [42]). It is easy to see that the coefficients $a_{i j}$ of equation (13) belong to the space of multipliers $M\left(W_{2}^{s-1}(\Omega)\right)$.

The following relations are valid [14]:

$$
\begin{aligned}
W_{2}^{|s-1|}(\Omega) & =M\left(W_{2}^{s-1}(\Omega)\right), & |s-1|>1, \\
W_{2 /|s-1|}^{|s-1|+\varepsilon}(\Omega) & \subset M\left(W_{2}^{s-1}(\Omega)\right), \quad \varepsilon>0, & 0<|s-1|<1, \\
L_{\infty}(\Omega) & =M\left(L_{2}(\Omega)\right)=M\left(W_{2}^{s-1}(\Omega)\right), & s=1 .
\end{aligned}
$$

Let us consider the FDS

$$
\mathcal{L}_{h} v \equiv-\frac{1}{2} \sum_{i, j=1}^{2}\left[\left(a_{i j} v_{x_{j}}\right)_{\bar{x}_{i}}+\left(a_{i j} v_{\bar{x}_{j}}\right)_{x_{i}}\right]=T_{1}^{2} T_{2}^{2} f, \quad x \in \omega_{h}
$$

with the previous boundary condition. The error $z=u-v$ satisfies the conditions

$$
\mathcal{L}_{h} z=\sum_{i, j=1}^{2} \eta_{i j, \bar{x}_{i}}, \quad x \in \omega_{h} ; \quad z=0, \quad x \in \gamma_{h}
$$

where $\eta_{i j}=T_{i}^{+} T_{3-i}^{2}\left(a_{i j} \frac{\partial u}{\partial x_{j}}\right)-\frac{1}{2}\left(a_{i j} u_{x_{j}}+a_{i j}^{+i} u_{\bar{x}_{j}}^{+i}\right)$. The following a priori estimates

$$
\begin{gathered}
\|z\|_{W_{2}^{2}\left(\omega_{h}\right)} \leqslant C \sum_{i, j=1}^{2}\left\|\eta_{i j, \bar{x}_{i}}\right\|_{L_{2}\left(\omega_{h}\right)}, \\
\|z\|_{W_{2}^{1}\left(\omega_{h}\right)} \leqslant C \sum_{i, j=1}^{2}\left\|\eta_{i j}\right\|_{L_{2}\left(\omega_{h}\right)}
\end{gathered}
$$

hold.

Using the bilinear version of the Bramble-Hilbert lemma or the interpolation properties of the bounded bilinear operators from (15) and (16), one obtains convergence rate estimates in the form (see $[14,16,19])$

$$
\begin{array}{lll}
\|z\|_{W_{2}^{2}\left(\omega_{h}\right)} \leqslant C h^{s-2} \max _{i, j}\left\|a_{i j}\right\|_{W_{2}^{s-1}(\Omega)}\|u\|_{W_{2}^{s}(\Omega)}, & & 2<s \leqslant 4, \\
\|z\|_{W_{2}^{1}\left(\omega_{h}\right)} \leqslant C h^{s-1} \max _{i, j}\left\|a_{i j}\right\|_{W_{2}^{s-1}(\Omega)}\|u\|_{W_{2}^{s}(\Omega)}, & & 2<s \leqslant 3, \\
\|z\|_{W_{2}^{1}\left(\omega_{h}\right)} \leqslant C h^{s-1} \max _{i, j}\left\|a_{i j}\right\|_{W_{2 /(s-1)}^{s-1+\varepsilon}(\Omega)}\|u\|_{W_{2}^{s}(\Omega)}, & & 1<s \leqslant 2 .
\end{array}
$$

Analogous results for the third boundary value problem were obtained in [26], for the problem with mixed boundary conditions in [4], and for one nonlocal BVP in [1]. Different error estimates for discrete methods approximating elliptic problems with variable coefficients were obtained in $[57,58]$. Further bibliographical references can be found in $[14,46]$.

In a multidimensional case $(n>2)$, additional problems caused by the discontinuity of right hand side of the equation $\left(f \in W_{2}^{s-2}(\Omega) \not \subset C(\bar{\Omega})\right.$ for $\left.s \leqslant 2+n / 2\right)$ or its solution $\left(u \in W_{2}^{s}(\Omega) \not \subset C(\bar{\Omega})\right.$ for $\left.s \leqslant n / 2\right)$ arise. These problems can be resolved by convenient averaging. Note also that $M\left(W_{2}^{s-1}(\Omega)\right) \neq W_{2}^{s-1}(\Omega)$ for $s \leqslant 1+n / 2$. 


\section{Equations with singular coefficients}

Interface problems occur in many physical applications. Such problems can be modelled by partial differential equations with singular coefficients. For example, as a model problem, let us consider the Dirichlet problem

$$
-\Delta u+c(x) \delta_{S}(x) u=f(x), \quad x \in \Omega ; \quad u=0, \quad x \in \Gamma,
$$

where $S$ is a continuous curve in $\Omega$ and $\delta_{S}(x)$ is Dirac's delta distribution [51] concentrated on $S$. We suppose that $c(x) \in L_{\infty}(S)$ and $0<C_{0} \leqslant c(x) \leqslant C_{1}$ almost everywhere on $S$.

We assume for simplicity that the curve $S$ divides $\Omega$ into two regions: $\bar{\Omega}=\bar{\Omega}_{1} \cup \bar{\Omega}_{2}$, $\Omega_{1} \cap \Omega_{2}=\emptyset$. Then, at some assumptions for smoothness, BVP (17) can be rewritten as follows:

$$
-\Delta u=f(x), \quad x \in \Omega_{1} \cup \Omega_{2} ; \quad[u]_{S}=0, \quad\left[\frac{\partial u}{\partial \nu}\right]_{S}=c(x) u,
$$

where $\partial u / \partial \nu$ - is the normal derivative.

We approximate BVP (17) on the mesh $\bar{\omega}_{h}$ with the following FDS (see [20]):

$$
-\Delta_{h} v+\alpha v=T_{1}^{2} T_{2}^{2} f \quad \text { in } \quad \omega_{h} ; \quad v=0 \quad \text { on } \quad \gamma_{h}
$$

where

$$
\begin{gathered}
\alpha(x)=T_{1}^{2} T_{2}^{2}\left(c \delta_{S}\right)= \begin{cases}h^{-2} \int_{S(x)} \kappa\left(x, x^{\prime}\right) c\left(x^{\prime}\right) d S_{x^{\prime}}, & x \in S_{h}, \\
0, & x \in \omega_{h} \backslash S_{h},\end{cases} \\
\kappa\left(x, x^{\prime}\right)=\left(1-\frac{\left|x_{1}^{\prime}-x_{1}\right|}{h}\right)\left(1-\frac{\left|x_{2}^{\prime}-x_{2}\right|}{h}\right), S(x)=S \cap e(x), e(x)=\left(x_{1}-h, x_{1}+h\right) \times\left(x_{2}-\right. \\
\left.h, x_{2}+h\right) \text { is the cell attached to the internal node } x \in \omega_{h}, \text { and } S_{h}=\left\{x \in \omega_{h}: S(x) \neq \emptyset\right\} .
\end{gathered}
$$

The error $z=u-v$ satisfies the FDS

$$
-\Delta_{h} z+\alpha z=-\psi_{1, \bar{x}_{1} x_{1}}-\psi_{2, \bar{x}_{2} x_{2}}+\chi \quad \text { in } \quad \omega_{h} ; \quad z=0 \quad \text { on } \quad \gamma_{h}
$$

where

$$
\begin{aligned}
& \psi_{i}=u-T_{3-i}^{2} u, \quad i=1,2, \\
& \chi=\alpha u-h^{-2} \int_{S(x)} \kappa\left(x, x^{\prime}\right) c\left(x^{\prime}\right) u\left(x^{\prime}\right) d S_{x^{\prime}}, \quad x \in S_{h}, \\
& \chi=0, \quad x \in \omega_{h} \backslash S_{h} .
\end{aligned}
$$

The a priori estimate

$$
\|z\|_{W_{2}^{1}\left(\omega_{h}\right)} \leqslant C\left[\left\|\psi_{1, x_{1}}\right\|_{L_{2}\left(\omega_{h}\right)}+\left\|\psi_{2, x_{2}}\right\|_{L_{2}\left(\omega_{h}\right)}+\left(h^{2} \sum_{x \in S_{h}} \frac{\chi^{2}}{\alpha}\right)^{1 / 2}\right]
$$

is satisfied. Estimating the terms in the right-hand side of (18) using the Bramble-Hilbert lemma, we obtain the following inequalities:

$$
\|z\|_{W_{2}^{1}\left(\omega_{h}\right)} \leqslant C h^{s-1}\|u\|_{W_{2}^{s}(\Omega)}, \quad 1<s<3 / 2
$$


and

$$
\|z\|_{W_{2}^{1}\left(\omega_{h}\right)} \leqslant C h^{s-1}\left(\|u\|_{W_{2}^{s}\left(\Omega_{1}\right)}+\|u\|_{W_{2}^{s}\left(\Omega_{2}\right)}+\|u\|_{W_{2}^{s-1 / 2}(\Omega)}\right), \quad 3 / 2 \leqslant s<2 .
$$

In the case where $S$ is a segment parallel to one of the coordinate axes, an improved convergence rate estimate holds. Let, for example, $S$ be given by the equation $x_{2}=j_{0} h$ $\left(j_{0}\right.$ - integer). Then

$\|z\|_{W_{2}^{1}\left(\omega_{h}\right)} \leqslant C h^{2}\left(\left\|\frac{\partial^{3} u}{\partial x_{1}^{2} \partial x_{2}}\right\|_{L_{2}(\Omega)}+\left\|\frac{\partial^{3} u}{\partial x_{1} \partial x_{2}^{2}}\right\|_{L_{2}\left(\Omega_{1}\right)}+\left\|\frac{\partial^{3} u}{\partial x_{1} \partial x_{2}^{2}}\right\|_{L_{2}\left(\Omega_{2}\right)}+\|c\|_{W_{2}^{2}(0,1)}\|u\|_{W_{2}^{2}(S)}\right)$.

\section{Parabolic problems}

In the parabolic case, analogous results hold. Let us consider the following initial-boundary value problem (IBVP):

$$
\begin{gathered}
\frac{\partial u}{\partial t}+\mathcal{L} u=f(x, t) \quad \text { in } \quad Q=\Omega \times(0, T) ; \\
u(x, 0)=u_{0}(x) ; \quad u(x, t)=0 \quad \text { on } \quad \Gamma \times(0, T) .
\end{gathered}
$$

Let us introduce the mesh $Q_{h \tau}=\omega_{h} \times \omega_{\tau}$, where $\omega_{\tau}$ is a uniform mesh with the step size $\tau$ in $(0, T)$. We also define the discrete $L_{2}-$ norm

$$
\|v\|_{L_{2}\left(Q_{h \tau}\right)}^{2}=h^{2} \tau \sum_{(x, t) \in Q_{h \tau}} v^{2}(x, t)
$$

and the discrete Sobolev norms $\|v\|_{W_{2}^{k, k / 2}\left(Q_{h \tau}\right)}$.

We consider the implicit FDS

$$
v_{\bar{t}}+\mathcal{L}_{h} v=T_{1}^{2} T_{2}^{2} T_{t}^{-} f
$$

with the corresponding boundary and initial conditions, where $T_{t}^{-}$is the Steklov averaging operator on $t$

$$
T_{t}^{-} f(x, t)=\frac{1}{\tau} \int_{t-\tau}^{t} f\left(x, t^{\prime}\right) d t^{\prime} .
$$

The error $z=u-v$ satisfies the equation

$$
z_{\bar{t}}+\mathcal{L}_{h} z=\varphi_{\bar{t}}+\sum_{i, j=1}^{2} \eta_{i j, \bar{x}_{i}}
$$

and homogeneous boundary and initial conditions. Here

$$
\varphi=u-T_{1}^{2} T_{2}^{2} u, \quad \eta_{i j}=T_{i}^{+} T_{3-i}^{2} T_{t}^{-}\left(a_{i j} \frac{\partial u}{\partial x_{j}}\right)-\frac{1}{2}\left(a_{i j} u_{x_{j}}+a_{i j}^{+i} u_{\bar{x}_{j}}^{+i}\right) .
$$

The following a priori estimates are valid:

$$
\|z\|_{W_{2}^{2,1}\left(Q_{h \tau}\right)} \leqslant C\left(\left\|\varphi_{\bar{t}}\right\|_{L_{2}\left(Q_{h \tau}\right)}+\sum_{i, j=1}^{2}\left\|\eta_{i j, \bar{x}_{i}}\right\|_{L_{2}\left(Q_{h \tau}\right)}\right)
$$


and

$$
\|z\|_{W_{2}^{1,1 / 2}\left(Q_{h \tau}\right)} \leqslant C\left([\varphi]_{1 / 2}+\sum_{i, j=1}^{2}\left\|\eta_{i j}\right\|_{L_{2}\left(Q_{h \tau}\right)}\right)
$$

where $[\varphi]_{1 / 2}^{2}=h^{2} \sum_{x \in \omega_{h}} \tau^{2} \sum_{t, t^{\prime} \in \bar{\omega}_{\tau}, t \neq t^{\prime}} \frac{\left|\varphi(x, t)-\varphi\left(x, t^{\prime}\right)\right|^{2}}{\left|t-t^{\prime}\right|^{2}}$. From here, in a similar manner as in the elliptic case, for $\tau \asymp h^{2}$, one obtains the convergence rate estimates (see $[10,11]$ )

$$
\|z\|_{W_{2}^{2,1}\left(Q_{h \tau}\right)} \leqslant C h^{s-2}\left(\max _{i, j}\left\|a_{i j}\right\|_{W_{2}^{s-1}(\Omega)}+1\right)\|u\|_{W_{2}^{s, s / 2}(Q)}
$$

for $2<s \leqslant 4$ and

$$
\|z\|_{W_{2}^{1,1 / 2}\left(Q_{h \tau}\right)} \leqslant C h^{s-1}\left(\max _{i, j}\left\|a_{i j}\right\|_{W_{2}^{s-1}(\Omega)}+\sqrt{\ln (1 / h)}\right)\|u\|_{W_{2}^{s, s / 2}(Q)}
$$

for $2<s \leqslant 3$. For $1 \leqslant s \leqslant 2$, the solution of IBVP (19) may be discontinuous. In this case, the error may be defined as $z=T_{1} T_{2} u-v$.

The problem with coefficients depending on $t$ was considered in [17]. Similar results were obtained for the FDS on non-uniform meshes (see $[22,23]$ ). FDSs on time adaptive meshes were considered in $[45,47]$. Convergence rate estimates in integral with respect to time norms were obtained in [48]. Sharp error estimates of vector splitting methods for the heat equation were derived in [54]. Note that some results in this area were obtained much earlier using the different technique (see, e.g., [32,55]). Further bibliographical references can be found in [14].

\section{Heat equation with concentrated capacity}

Let us consider the IBVP for the heat equation with the presence of concentrated capacity at interior point $x=\xi[39]$

$$
\begin{gathered}
{[c(x)+K \delta(x-\xi)] \frac{\partial u}{\partial t}-\frac{\partial}{\partial x}\left(a(x) \frac{\partial u}{\partial x}\right)=f(x, t), \quad(x, t) \in Q,} \\
u(0, t)=0, \quad u(1, t)=0, \quad 0<t<T \\
u(x, 0)=u_{0}(x), \quad x \in(0,1),
\end{gathered}
$$

where $Q=(0,1) \times(0, T), K>0,0<c_{1} \leqslant a(x) \leqslant c_{2}, 0<c_{3} \leqslant c(x) \leqslant c_{4}$ and $\delta(x)$ is the Dirac distribution. The solution of $\operatorname{IBVP}(20)$ satisfies the equation

$$
c(x) \frac{\partial u}{\partial t}-\frac{\partial}{\partial x}\left(a(x) \frac{\partial u}{\partial x}\right)=f(x, t)
$$

for $(x, t) \in Q_{1}=(0, \xi) \times(0, T)$ and $(x, t) \in Q_{2}=(\xi, 1) \times(0, T)$, while for $x=\xi$ the conjugation conditions

$$
[u]_{x=\xi} \equiv u(\xi+0, t)-u(\xi-0, t)=0, \quad\left[a \frac{\partial u}{\partial x}\right]_{x=\xi}=K \frac{\partial u(\xi, t)}{\partial t}
$$

are fulfilled. 
We introduce the space $\widetilde{L}_{2}(0,1)=\widetilde{W}_{2}^{0}(0,1)$ of functions $w(x) \in L_{2}(0,1)$ equipped with an inner product and norm

$$
(u, w)_{\widetilde{L}_{2}(0,1)}=\int_{0}^{1} u(x) w(x) d x+u(\xi) w(\xi), \quad\|w\|_{\widetilde{L}_{2}(0,1)}=(u, w)_{\widetilde{L}_{2}(0,1)}^{1 / 2} .
$$

Further we set $\widetilde{W_{2}^{1}}(0,1)=\stackrel{\circ}{W_{2}^{1}}(0,1)$ and $\widetilde{W}_{2}^{k}(0,1)=\stackrel{\circ}{W_{2}^{1}}(0,1) \cap W_{2}^{k}(0, \xi) \cap W_{2}^{k}(\xi, 1), k=$ $2,3, \ldots$ We also define the spaces $\widetilde{W}_{2}^{k, k / 2}(Q)=L_{2}\left(0, T ; \widetilde{W}_{2}^{k}(0,1)\right) \cap W_{2}^{k / 2}\left(0, T ; \widetilde{L}_{2}(0,1)\right)$, $k=0,1,2, \ldots$

Let $\omega_{h}$ be a uniform mesh in $(0,1)$ with the step-size $h$. For simplicity, we assume that $\xi \in \omega_{h}$. We approximate IBVP $(20)$ on the mesh $Q_{h \tau}=\omega_{h} \times \omega_{\tau}$ by an implicit FDS with an averaged right hand side (see $[29,30]$ )

$$
\begin{gathered}
\left(c+K \delta_{h}\right) v_{\bar{t}}-\left(\tilde{a} v_{\bar{x}}\right)_{x}=T_{x}^{2} T_{t}^{-} f, \quad(x, t) \in Q_{h \tau}, \\
v(0, t)=0, \quad v(1, t)=0, \quad t \in \omega_{\tau}, \\
v(x, 0)=u_{0}(x), \quad x \in \bar{\omega}_{h},
\end{gathered}
$$

where $\tilde{a}(x)=[a(x-0)+a(x-h+0)] / 2, \delta_{h}=\delta_{h}(x-\xi)=\left\{\begin{array}{ll}0, & x \in \omega_{h} \backslash\{\xi\} \\ 1 / h, & x=\xi\end{array}\right.$ is the mesh Dirac function and $T_{x}$ is the Steklov averaging operator on variable $x$.

Let $(v, w)_{h}$ be a discrete $L_{2}$-inner product in $\omega_{h}$. Let us set $B_{h} w=\left(1+\delta_{h}\right) w$ and define the energy norms $\|w\|_{B_{h}}=\left(B_{h} w, w\right)_{h}^{1 / 2}$ and $\|w\|_{B_{h}^{-1}}=\left(B_{h}^{-1} w, w\right)_{h}^{1 / 2}$. We introduce the mesh Sobolev norms with the weight operator $B_{h}$ :

$$
\begin{gathered}
\|w\|_{\widetilde{L}_{2}\left(\omega_{h}\right)}^{2}=\|w\|_{B_{h}}^{2}=\|w\|_{L_{2}\left(\omega_{h}\right)}^{2}+w^{2}(\xi), \quad\|w\|_{\widetilde{W}_{2}^{1}\left(\omega_{h}\right)}^{2}=\left\|w_{\bar{x}}\right\|_{L_{2}\left(\omega_{h}\right)}^{2}+\|w\|_{\widetilde{L}_{2}\left(\omega_{h}\right)}^{2}, \\
\|w\|_{\widetilde{W}_{2}^{2}\left(\omega_{h}\right)}^{2}=\left\|w_{\bar{x} x}\right\|_{B_{h}^{-1}}^{2}+\|w\|_{\widetilde{W}_{2}^{1}\left(\omega_{h}\right)}^{2} ; \quad\|w\|_{\widetilde{L}_{2}\left(Q_{h \tau}\right)}^{2}=\tau \sum_{t \in \omega_{\tau}}\|w(\cdot, t)\|_{\widetilde{L}_{2}\left(\omega_{h}\right)}^{2}, \\
\|w\|_{\widetilde{W}_{2}^{1,1 / 2}\left(Q_{h \tau}\right)}^{2}=\tau \sum_{t \in \bar{\omega}_{\tau}}\|w(\cdot, t)\|_{\widetilde{W}_{2}^{1}\left(\omega_{h}\right)}^{2}+\tau^{2} \sum_{t \in \bar{\omega}_{\tau}} \sum_{t^{\prime} \in \bar{\omega}_{\tau}, t^{\prime} \neq t} \frac{\left\|w(\cdot, t)-w\left(\cdot, t^{\prime}\right)\right\|_{B_{h}}^{2}}{\left|t-t^{\prime}\right|^{2}} \\
\|w\|_{\widetilde{W}_{2}^{2,1}\left(Q_{h \tau}\right)}^{2}=\tau \sum_{t \in \bar{\omega}_{\tau}}\|w(\cdot, t)\|_{\widetilde{W}_{2}^{2}\left(\omega_{h}\right)}^{2}+\tau \sum_{t \in \omega_{\tau}}\left\|w_{\bar{t}}(\cdot, t)\right\|_{B_{h}}^{2} .
\end{gathered}
$$

The error $z=u-v$ satisfies the following conditions:

$$
\begin{gathered}
\left(c+K \delta_{h}\right) z_{\bar{t}}-\left(\tilde{a} z_{\bar{x}}\right)_{\hat{x}}=\varphi, \quad(x, t) \in Q_{h \tau}, \\
z(0, t)=0, \quad z(1, t)=0, \quad t \in \omega_{\tau}, \\
z(x, 0)=0, \quad x \in \bar{\omega}_{h},
\end{gathered}
$$

where $\varphi=\psi_{\bar{t}}-\chi_{x}, \psi=c u-T_{x}^{2}(c u)$ and $\chi=\tilde{a} u_{\bar{x}}-T_{x}^{-} T_{t}^{-}\left(a \frac{\partial u}{\partial x}\right)$. The following a priori estimates hold:

$$
\|z\|_{\widetilde{W}_{2}^{2,1}\left(Q_{h \tau}\right)} \leqslant C\left(\tau \sum_{t \in \omega_{\tau}}\|\varphi(\cdot, t)\|_{B_{h}^{-1}}^{2}\right)^{1 / 2}
$$




$$
\begin{aligned}
\|z\|_{\widetilde{W}_{2}^{1,1 / 2}\left(Q_{h \tau}\right)} \leqslant & C\left[\tau^{2} \sum_{t \in \bar{\omega}_{\tau}} \sum_{t^{\prime} \in \bar{\omega}_{\tau}, t^{\prime} \neq t} \frac{\left\|\psi(\cdot, t)-\psi\left(\cdot, t^{\prime}\right)\right\|_{B_{h}^{-1}}^{2}}{\left|t-t^{\prime}\right|^{2}}\right. \\
& \left.+\tau \sum_{t \in \omega_{\tau}}\left(\frac{1}{t}+\frac{1}{T-t}\right)\|\psi(\cdot, t)\|_{B_{h}^{-1}}^{2}+\tau \sum_{t \in \omega_{\tau}}\|\chi(\cdot, t)\|_{L_{2}\left(\omega_{h}\right)}^{2}\right]^{1 / 2} .
\end{aligned}
$$

Using integral representations of $\psi$ and $\chi$ and the form of the corresponding norms, from (21) and (22) we obtain the following convergence rate estimates:

$$
\begin{gathered}
\|z\|_{\widetilde{W}_{2}^{2,1}\left(Q_{h \tau}\right)} \leqslant C\left(h^{2}+\tau\right)\left(\|a\|_{W_{2}^{3}(0, \xi)}+\|a\|_{W_{2}^{3}(\xi, 1)}+\|c\|_{W_{2}^{2}(0,1)}\right)\|u\|_{\widetilde{W}_{2}^{4,2}(Q)} \cdot \\
\|z\|_{\widetilde{W}_{2}^{1,1 / 2}\left(Q_{h \tau}\right)} \leqslant C\left(h^{2} \sqrt{\ln 1 / \tau}+\tau\right)\left(\|a\|_{W_{2}^{2}(0, \xi)}+\|a\|_{W_{2}^{2}(\xi, 1)}+\|c\|_{W_{2}^{2}(0,1)}\right)\|u\|_{\widetilde{W}_{2}^{3,3 / 2}(Q)} .
\end{gathered}
$$

Similar estimates can be obtained in the norm $\widetilde{L}_{2}\left(Q_{h \tau}\right)$ using an appropriate approximation of the initial condition. FDSs on nonuniform meshes are considered in [29].

\section{Hyperbolic problems}

Convergence rate estimates for hyperbolic IBVPs, contrary to the case of elliptic and parabolic problems, are usually inconsistent with the smoothness of data. Let us consider the following IBVP:

$$
\begin{aligned}
& \frac{\partial^{2} u}{\partial t^{2}}+\mathcal{L} u=f(x, t) \quad \text { in } \quad Q=\Omega \times(0, T)=(0,1)^{2} \times(0, T) ; \\
& u(x, 0)=u_{0}(x), \quad \frac{\partial u(x, 0)}{\partial t}=u_{1}(x) ; \quad u(x, t)=0 \text { on } \Gamma \times(0, T) .
\end{aligned}
$$

We introduce the mesh in the same manner as in Section 7 and define the norm

$$
\|v\|_{C_{\tau}\left(W_{2}^{1}\left(\omega_{h}\right)\right)}=\max _{t \in \omega_{\tau}}\left[\left\|v_{\bar{t}}\right\|_{L_{2}\left(\omega_{h}\right)}^{2}+\sum_{i=1}^{2}\left\|\left(\frac{v+v^{-}}{2}\right)_{x_{i}}\right\|_{L_{2}\left(\omega_{h}\right)}^{2}\right]^{1 / 2} .
$$

Consider the FDS

$$
v_{t \bar{t}}+\frac{1}{4} \mathcal{L}_{h}\left(v^{+}+2 v+v^{-}\right)=T_{1} T_{2} T_{t} f
$$

where $v^{ \pm}=v(x, t \pm \tau)$, with the corresponding initial and boundary conditions. For $\tau \asymp h$ the following convergence rate estimate is valid (see $[12,18]$ ):

$$
\|z\|_{C_{\tau}\left(W_{2}^{1}\left(\omega_{h}\right)\right)} \leqslant C h^{s-2}\left(\max _{i, j}\left\|a_{i j}\right\|_{W_{2}^{s-1}(\Omega)}+1\right)\|u\|_{W_{2}^{s}(Q)}
$$

for $2<s \leqslant 4$.

In some cases, by the interpolation technique one can obtain estimates which guarantee a faster convergence on weaker solutions (see $[56,57]$ ). Let us consider the following model problem:

$$
\begin{gathered}
\frac{\partial^{2} u}{\partial t^{2}}=\frac{\partial^{2} u}{\partial x^{2}} \quad \text { in } \quad Q=(0,1) \times(0, T) ; \\
u(x, 0)=u_{0}(x), \quad \frac{\partial u(x, 0)}{\partial t}=0 ; \quad u(x, t)=0 \text { on }\{0,1\} \times(0, T)
\end{gathered}
$$


and approximate it by an FDS of the form of (24). Using the integral representation of the residual, one easily obtains the estimates

$$
\|z\|_{C_{\tau}\left(W_{2}^{1}\left(\omega_{h}\right)\right)} \leqslant C(h+\tau)^{2}\left\|u_{0}\right\|_{W_{2}^{4}(0,1)}, \quad\|z\|_{C_{\tau}\left(W_{2}^{1}\left(\omega_{h}\right)\right)} \leqslant C\left\|u_{0}\right\|_{W_{2}^{1}(0,1)} .
$$

From these estimates by interpolation one obtains [13]

$$
\|z\|_{C_{\tau}\left(W_{2}^{1}\left(\omega_{h}\right)\right)} \leqslant C(h+\tau)^{\frac{2}{3}(s-1)}\left\|u_{0}\right\|_{W_{2}^{s}(0,1)}, \quad 1 \leqslant s \leqslant 4 .
$$

Contrary to (25), estimate (26) guarantees convergence even for $1<s \leqslant 2$.

For further bibliographical references see [14].

\section{String equation with a concentrated mass}

Let us consider the first IBVP for the equation of a vibrating string with a concentrated mass at the interior point $x=\xi[50]$

$$
\begin{gathered}
{[c(x)+K \delta(x-\xi)] \frac{\partial^{2} u}{\partial t^{2}}-\frac{\partial}{\partial x}\left(a(x) \frac{\partial u}{\partial x}\right)=f(x, t), \quad(x, t) \in Q=(0,1) \times(0, T),} \\
u(0, t)=0, \quad u(1, t)=0, \quad 0<t<T \\
u(x, 0)=u_{0}(x), \quad \frac{\partial u(x, 0)}{\partial t}=u_{1}(x), \quad x \in(0,1),
\end{gathered}
$$

where $a(x), c(x)$ and $K$ are the same as in Section 8. Keeping notations from Section 8, we approximate IBVP (27) by a symmetric weighted difference scheme with an averaged right-hand side (see $[28,31])$

$$
\begin{gathered}
\left(c+K \delta_{h}\right) v_{t \bar{t}}-\left(\tilde{a} v_{\bar{x}}^{(\sigma)}\right)_{x}=T_{x}^{2} T_{t}^{2} f, \quad(x, t) \in Q_{h \tau}, \\
v(0, t)=0, \quad v(1, t)=0, \quad t \in \bar{\omega}_{\tau} ; \quad v(x, 0)=u_{0}(x), \quad x \in \omega_{h}, \\
\left(c+K \delta_{h}\right) v_{t}(x, 0)=T_{x}^{2}\left(c u_{1}\right)+K \delta_{h} u_{1}+\frac{\tau}{2} T_{x}^{2}\left[\widetilde{T}_{t}^{2} f(x, 0)+\left(a u_{0}^{\prime}(x)\right)^{\prime}\right], \quad x \in \omega_{h},
\end{gathered}
$$

where $v^{(\sigma)}=\sigma v^{+}+(1-2 \sigma) v+\sigma v^{-}, \sigma \geqslant 1 / 4$ and

$$
\widetilde{T}_{t}^{2} f(x, 0)=\frac{2}{\tau} \int_{0}^{\tau}\left(1-\frac{t^{\prime}}{\tau}\right) f\left(x, t^{\prime}\right) d t^{\prime}
$$

The error $z=u-v$ satisfies the a priori estimate

$$
\|z\|_{C_{\tau}\left(\widetilde{L}_{2}\left(\omega_{h}\right)\right)}^{2} \leqslant C\left[\|\chi\|_{L_{2}\left(\omega_{h}\right)}^{2}+\tau^{2}\left\|\chi_{x}\right\|_{B_{h}^{-1}}^{2}+\tau \sum_{t \in \omega_{\tau}}\|\varphi(\cdot, t)\|_{L_{2}\left(\omega_{h}\right)}^{2}+\tau \sum_{t \in \omega_{\tau}}\left\|\eta_{t}(\cdot, t)\right\|_{B_{h}^{-1}}^{2}\right]
$$

where

$$
\begin{gathered}
\varphi=T_{x}^{-} T_{t}^{2}\left(a \frac{\partial u}{\partial x}\right)-\tilde{a} u_{\bar{x}}^{(\sigma)}, \quad \eta=c u-T_{x}^{2}(c u), \\
\chi=\left.\frac{\tau}{2} T_{x}^{-}\left[a\left(\widetilde{T}_{t}^{2} \frac{\partial u}{\partial x}-\frac{d u_{0}}{d x}\right)\right]\right|_{t=0},
\end{gathered}
$$


and

$$
\|z\|_{C_{\tau}\left(\widetilde{L}_{2}\left(\omega_{h}\right)\right)}=\max _{t \in \omega_{\tau}}\left\|\left(z+z^{-}\right) / 2\right\|_{\widetilde{L}_{2}\left(\omega_{h}\right)} .
$$

Estimating the terms $\chi, \varphi$ and $\eta$ similarly to the previous cases, we obtain the following convergence rate estimate:

$$
\|z\|_{C_{\tau}\left(\widetilde{L}_{2}\left(\omega_{h}\right)\right)} \leqslant C\left(h^{2}+\tau^{2}\right)\left(\|a\|_{W_{2}^{2}(0, \xi)}+\|a\|_{W_{2}^{2}(\xi, 1)}+\|c\|_{W_{2}^{2}(0,1)}\right)\|u\|_{\widetilde{W}_{2}^{3}(Q)} .
$$

Here the space $\widetilde{W}_{2}^{k}(Q)(k=0,1,2, \ldots)$ is defined as the closure of the subset of functions $w \in L_{2}(Q)$ such that

$$
\begin{aligned}
w(0, t) & =w(1, t)=0, & \frac{\partial^{i} w}{\partial t^{i}} & \in L_{2}\left(0, T ; \widetilde{L}_{2}(0,1)\right), \quad i=0,1, \ldots, k, \\
\frac{\partial^{i} w}{\partial x \partial t^{i-1}} & \in L_{2}(Q), i=1,2, \ldots, k, & \frac{\partial^{i} w}{\partial x^{j} \partial t^{i-j}} & \in L_{2}\left(Q_{1}\right) \cap L_{2}\left(Q_{2}\right), 2 \leqslant j \leqslant k, i=j, \ldots, k
\end{aligned}
$$

in the norm

$$
\begin{aligned}
\|w\|_{\widetilde{W}_{2}^{k}(Q)}^{2}= & \sum_{i=0}^{k}\left(\left\|\frac{\partial^{i} w(\xi, \cdot)}{\partial t^{i}}\right\|_{L_{2}(0, T)}^{2}+\left\|\frac{\partial^{i} w}{\partial t^{i}}\right\|_{L_{2}(Q)}^{2}\right)+\sum_{i=1}^{k}\left\|\frac{\partial^{i} w}{\partial x \partial t^{i-1}}\right\|_{L_{2}(Q)}^{2} \\
& +\sum_{j=2}^{k} \sum_{i=j}^{k}\left(\left\|\frac{\partial^{i} w}{\partial x^{j} \partial t^{i-j}}\right\|_{L_{2}\left(Q_{1}\right)}^{2}+\left\|\frac{\partial^{i} w}{\partial x^{j} \partial t^{i-j}}\right\|_{L_{2}\left(Q_{2}\right)}^{2}\right) .
\end{aligned}
$$

A similar result in the norm

$$
\|z\|_{C_{\tau}\left(\widetilde{W}_{2}^{1}\left(\omega_{h}\right)\right)}=\max _{t \in \omega_{\tau}}\left[\left\|\left(z+z^{-}\right) / 2\right\|_{\widetilde{W}_{2}^{1}\left(\omega_{h}\right)}^{2}+\left\|z_{t}\right\|_{\widetilde{L}_{2}\left(\omega_{h}\right)}^{2}\right]^{1 / 2}
$$

was obtained in $[28,31]$.

\section{Nonlinear problems}

Analogous results can be obtained for some nonlinear BVPs. Let us consider, as the model problem, the first IBVP for the quasilinear heat equation

$$
\begin{gathered}
\frac{\partial u}{\partial t}-\frac{\partial}{\partial x}\left(a(u) \frac{\partial u}{\partial x}\right)=f(x, t), \quad(x, t) \in Q=(0,1) \times(0, T) \\
u(0, t)=0, \quad u(1, t)=0, \quad 0<t<T \\
u(x, 0)=u_{0}(x), \quad x \in(0,1) .
\end{gathered}
$$

Introduce the range $\mathcal{M}_{u}$ of exact solutions and define the neighborhood $\mathcal{D}_{u}$ of this range

$$
\mathcal{M}_{u}=\left\{u: u_{1} \leqslant u \leqslant u_{2}\right\}, \quad \mathcal{D}_{u}=\left\{\tilde{u}: u_{1}-\delta \leqslant \tilde{u} \leqslant u_{2}+\delta\right\}, \quad \delta=\text { const }>0 .
$$

Suppose problem $(28)$ is uniquely solvable in $W_{2}^{2,1}(Q), u_{0} \in W_{2}^{1}(0,1)$ and $a \in C\left(\mathcal{D}_{u}\right)$, $0<k_{1} \leqslant a(v) \leqslant k_{2}$ for $v \in \mathcal{D}_{u}$.

Define the new function $\phi(u)=\int_{0}^{u} a(w) d w$ and reduce equation (20) to the form

$$
\frac{\partial u}{\partial t}-\frac{\partial^{2} \phi(u)}{\partial x^{2}}=f(x, t), \quad(x, t) \in Q
$$


Let us introduce a uniform mesh $Q_{h \tau}$ in the same way as in Section 8 and approximate IBVP (28), (29) by the explicit FDS

$$
\begin{gathered}
v_{t}-(\phi(v))_{\bar{x} x}=T_{x}^{2} T_{t} f, \quad(x, t) \in Q_{h \tau}, \\
v(0, t)=0, \quad v(1, t)=0, \quad t \in \omega_{\tau}, \quad v(x, 0)=T_{x}^{2} u_{0}(x), \quad x \in \bar{\omega}_{h} .
\end{gathered}
$$

For the error $z$ defined as $z=\bar{u}-v=T_{x}^{2} u-v$, we obtain the following FDS:

$$
\begin{gathered}
z_{t}-[\phi(\bar{u})-\phi(\bar{u}-z)]_{\bar{x} x}=\eta_{\bar{x} x}, \quad(x, t) \in Q_{h \tau}, \quad \eta=T_{t} \phi(u)-\phi\left(T_{x}^{2} u\right), \\
z(0, t)=0, \quad z(1, t)=0, \quad t \in \omega_{\tau}, \quad z(x, 0)=0, \quad x \in \bar{\omega}_{h} .
\end{gathered}
$$

Suppose the condition $\tau \leqslant k_{1} h^{2} /\left(16 k_{2}^{2}\right)$ is satisfied. Then for sufficiently small $h \leqslant h_{0}$ and $\tau \leqslant \tau_{0}$ there exists a unique solution of FDS (30) that converges to the solution of IBVP (28), (29) as $h \rightarrow 0, \tau \rightarrow 0$ and

$$
\max _{(x, t) \in Q_{h \tau}}|z(x, t)| \leqslant C \frac{h^{2}+\tau}{h^{3 / 2}}\|a\|_{C\left(\mathcal{D}_{u}\right)}\|u\|_{W_{2}^{2,1}(Q)} \leqslant \delta .
$$

Also, the a priori estimate

$$
\|z\|_{L_{2}\left(Q_{h \tau}\right)} \leqslant C\|\eta\|_{L_{2}\left(Q_{h \tau}\right)}
$$

holds. Using the Bramble-Hilbert lemma, one obtains the following convergence rate estimates (see $[24,25])$ :

$$
\begin{gathered}
\|v-\bar{u}\|_{L_{2}\left(Q_{h \tau}\right)} \leqslant C\left(h^{2}+\tau\right)\|a\|_{C\left(\mathcal{D}_{u}\right)}\|u\|_{W_{2}^{2,1}(Q)}, \\
\|v-u\|_{L_{2}\left(Q_{h \tau}\right)} \leqslant C\left(h^{2}+\tau\right)\left(\|a\|_{C\left(\mathcal{D}_{u}\right)}+1\right)\|u\|_{W_{2}^{2,1}(Q)} .
\end{gathered}
$$

A similar result for a stationary quasilinear BVP was obtained in [41].

\section{Acknowledgements}

Author wishes to thank prof. P. P. Matus for valuable comments and suggestions. This work has been supported by Ministry of Science, Technologies and Development of Republic of Serbia under grant 1645.

\section{References}

[1] G. Berikelashvili, On the convergence of the finite-difference scheme for one nonlocal elliptic boundary value problem, Publ. Inst. Math., 70 (84) (2001), pp. 69-78.

[2] G. Berikelashvili, On a nonlocal boundary value problem for the two-dimensional elliptic equation, Comput. Methods Appl. Math., 3 (2003), pp. 35-44.

[3] J.H. Bramble and S. R. Hilbert, Bounds for a class of linear functionals with application to Hermite interpolation, Numer. Math., 16 (1971), pp. 362-369.

[4] M. D. Chkhartishvili, G. Berikelashvili, On the convergence in $W_{2}^{1}$ of difference solutions for an elliptic equation with mixed boundary conditions, Bull. Acad. Sci. Georgia, 148 (1993), No. 2, pp. 180-184 (in Russian).

[5] P. G. Ciarlet, The Finite Element Method for Elliptic Problems, North-Holland, Amsterdam, 1978. 
[6] N. T. Drenska, Convergence of finite element scheme for the Poisson equation in $L_{p}-n o r m$, Vest. Mosk. Univ. Ser. XV, 3 (1984), pp. 19-22 (in Russian).

[7] T. Dupont and R. Scott, Polynomial approximation of functions in Sobolev spaces, Math. Comput., 34 (1980), pp. 441-463.

[8] I.P. Gavrilyuk, R.D. Lazarov, V.L. Makarov and S.I. Pirnazarov, Convergence rate estimates for finite difference schemes for elliptic equations of fourth order, Zh. Vychisl. Mat. Mat. Fiz., 23 (1983), pp. 355-365 (in Russian).

[9] B.S. Jovanović, On the convergence of discrete solutions to generalized solutions of boundary value problems, In: N. S. Bakhvalov, Yu. A. Kuznetsov (eds.), Variational-difference methods in mathematical physics, Proc. conf. held in Moscow 1983, OVM AN SSSR, Moscow, 1984, pp. 120-129 (in Russian).

[10] B.S. Jovanović, On the convergence of finite difference schemes for parabolic equations with variable coefficients, Numer. Math., 54 (1989), pp. 395-404.

[11] B.S. Jovanović, Convergence of finite-difference schemes for parabolic equations with variable coefficients, Z. Angew. Math. Mech., 71 (1991), No. 6, pp. 647-650.

[12] B.S. Jovanović, Convergence of finite-difference schemes for hyperbolic equations with variable coeffcients, Z. Angew. Math. Mech., 72 (1992), No. 6, pp. 493-496.

[13] B.S. Jovanović, On the estimates of the convergence rate of the finite difference schemes for the approximation of solutions of hyperbolic problems, Publ. Inst. Math., 52 (66) (1992), pp. 127-135.

[14] B.S. Jovanović, The finite difference method for boundary value problems with weak solutions, Posebna Izd., Mat. Inst., Beograd, No. 16, 1993.

[15] B. S. Jovanović, Interpolation of function spaces and the convergence rate estimates for the finite difference schemes, In: 2nd Int. Coll. on Numerical Analysis held in Plovdiv 1993, (D. Bainov and V. Covachev, eds.), VPS, Utrecht, 1994, pp. 103-112.

[16] B.S. Jovanović, Interpolation technique and convergence rate estimates for finite difference method, Lect. Notes Comput. Sci., 1196 (1997), pp. 200-211.

[17] B.S. Jovanović and D. Bojović, Finite difference method for the heat equation with coefficient from anisotropic Sobolev space, Facta Univ., Ser. Math. Inf., 15 (2000), pp. 113-122.

[18] B. S. Jovanović, L. D. Ivanović and E. E. Süli, Convergence of a finite-difference scheme for second-order hyperbolic equations with variable coefficients, IMA J. Numer. Anal., 7 (1987), pp. 39-45.

[19] B.S. Jovanović, L.D. Ivanović and E. E. Süli, Convergence of finite-difference schemes for elliptic equations with variable coefficients, IMA J. Numer. Anal., 7 (1987), pp. 301-305.

[20] B. S. Jovanović, J. D. Kandilarov and L. G. Vulkov, Construction and convergence of difference schemes for a model elliptic equation with Dirac's delta function coefficient, Lect. Notes Comput. Sci. 1988 (2001), pp. 431-438.

[21] B.S. Jovanovich and P.P. Matus, Estimation of the convergence rate of finite-difference schemes for elliptic problems, Zh. Vychisl. Mat. Mat. Fiz., 39 (1999), pp. 61-69 (in Russian).

$[22]$ B. S. Jovanovich, P.P. Matus and V.S. Shcheglik, Finite-difference schemes on nonuniform meshes for parabolic equation with variable coefficients and weak solutions, Dokl. NAN Belarusi, 42 (1998), No. 6, pp. 38-44 (in Russian).

[23] B.S. Jovanovich, P.P. Matus and V.S. Shcheglik, The rates of convergence of the finite-difference schemes on nonuniform meshes for parabolic equation with variable coefficients and weak solutions, Sib. Zh. Vychisl. Mat., 2 (1999), pp. 123-136 (in Russian).

[24] B.S. Jovanovich, P.P. Matus and V.S. Shcheglik, On accuracy of difference schemes for nonlinear parabolic equations with generalized solutions, Zh. Vychisl. Mat. Mat. Fiz., 39 (1999), pp. 1679-1686 (in Russian).

[25] B. S. Jovanovich, P. P. Matus and V.S. Shcheglik, Difference schemes for nonlinear parabolic equations with generalized solutions, Dokl. NAN Belarusi, 43 (1999), No. 5, pp. 25-29 (in Russian). 
[26] B.S. Jovanović and B.Z. Popović, Convergence of a finite difference scheme for the third boundary value problem for elliptic equation with variable coefficients, Comput. Methods Appl. Math., 1 (2001), No. 4, pp. 356-366.

[27] B.S. Jovanović, E. E. Süli and L. D. Ivanović, On finite difference schemes of high order accuracy for elliptic equations with mixed derivatives, Mat. Vesn., 38 (1986), pp. 131-136.

[28] B.S. Jovanović and L. G. Vulkov, On the convergence of difference schemes for the string equation with concentrated mass, In: R. Ciegis, A. Samarskii and M. Sapagovas (eds.), Finite-Difference Schemes: Theory and Applications, Proc of 3rd Int. Conf. held in Palanga (Lithuania) 2000, IMI, Vilnius, 2000, pp. 107-116.

[29] B.S. Jovanović and L. G. Vulkov, Operator's approach to the problems with concentrated factors, Lect. Notes Comput. Sci., 1988 (2001), pp. 439-450.

[30] B.S. Jovanović and L. G. Vulkov, On the convergence of finite difference schemes for the heat equation with concentrated capacity, Numer. Math., 89 (2001), No. 4, pp. 715-734.

[31] B. S. Jovanović and L. G. Vulkov, On the convergence of difference schemes for hyperbolic problems with concentrated data, SIAM J. Numer. Anal., 41 (2003), No. 2, pp. 516-538.

[32] M. L. Junkosa and D. M. Young, On the order of convergence of solutions of a difference equation to a solution of the diffusion equation, SIAM J., 1 (1953), pp. 111-135.

[33] V.M. Kalinin and V.L. Makarov, Convergence rate estimate in $L_{2}$-norm for difference scheme approximating third boundary value problem for elasticity theory on solutions from $W_{2}^{1}(\Omega)$ in axially symmetric case, Differ. Uravn., 23 (1987), pp. 1207-1219 (in Russian).

[34] R.D. Lazarov, On the convergence of finite-difference schemes on generalized solutions of Poisson equation, Differ. Uravn., 17 (1981), pp. 1285-1294 (in Russian).

[35] R. D. Lazarov, On the convergence of finite-difference schemes on generalized solutions of biharmonic equation in rectangle, Differ. Uravn., 17 (1981), pp. 1295-1303 (in Russian).

[36] R. D. Lazarov, V. L. Makarov, Difference schemes for axially symmetric Poisson equation with generalized solutions, Zh. Vychisl. Mat. Mat. Fiz., 21 (1981), pp. 1168-1180 (in Russian).

[37] R. D. Lazarov, V. L. Makarov and A. A. Samarskiŭ, Application of exact difference schemes for construction and investigation of difference schemes for generalized solutions, Mat. Sb., 117 (1982), pp. 469-480 (in Russian).

[38] J. L. Lions and E. Magenes, Problèmes aux Limites non Homogènes et Applications, Dunod, Paris, 1968.

[39] A. V. Lykov, Heat and Mass Transfer, Nauka, Moscow, 1989 (in Russian).

[40] V.L. Makarov and A.I. Ryzhenko, Consistent convergence rate estimates for finite difference scheme for axially symmetric Poisson equation in spheric coordinates, Zh. Vychisl. Mat. Mat. Fiz., 27 (1987), pp. 1252-1255 (in Russian).

[41] P. P. Matus, M. N. Moskal'kov and V.S. Shcheglik, Consistent convergence rate estimates for difference schemes for second order nonlinear parabolic equation with generalized solutions, Differ. Uravn., 31 (1995), pp. 1219-1226 (in Russian).

[42] V. G. Maz'ya and T. O. Shaposhnikova, Theory of multipliers in spaces of diferentiable functions, Monographs and Studies in Mathematics, 23 (1985), Pitman, Boston, Mass..

[43] Yu. I. Mokin, Discrete analogue of theorem on multiplier, Zh. Vychisl. Mat. Mat. Fiz., 11 (1971), pp. 746-749 (in Russian).

[44] A. A. Samarskii, Theory of Difference Schemes. Nauka, Moscow, 1983 (in Russian).

[45] A. A. Samarskii, B. S. Jovanovich, P. P. Matus and V.S. Shcheglik, Finite-difference schemes on adaptive time grids for parabolic equations with generalized solutions, Differ. Uravn., 33 (1997), pp. 975-984.

[46] A. A. Samarskii, R. D. Lazarov and V.L. Makarov, Finite-difference Schemes for Difference Equations with Generalized Solutions, Vysshaya shkola, Moscow, 1987 (in Russian). 
[47] A. A. Samarskii, P.P. Matus and P.N. Vabishchevich, Difference Schemes with Operator Factors, Kluwer, Boston, Dordrecht, London, 2002.

[48] A. A. Samarskii, P.P. Matus and P. N. Vabishchevich, Stability and convergence of two-level difference schemes in integral with respect to time norms, Math. Models Methods Appl. Sci., 8 (1998), No. 6, pp. $1055-1070$.

[49] E. E. Süli, B. S. Jovanović and L. D. Ivanović, Finite difference approximations of generalized solutions, Math. Comput., 45 (1985), pp. 319-327.

[50] A. N. Tikhonov and A. A. Samarskiǔ, Equations of Mathematical Physics, GITTL, Moscow, 1953 (in Russian).

[51] V.S. Vladimirov, Equations of Mathematical Physics, Nauka, Moscow, 1998 (in Russian).

[52] S. A. Voitsekhovskiu and V.N. Novichenko, Foundation of finite difference scheme of higher order for Dirichlet problem for Poisson equation with generalized solution, Differ. Uravn., 24 (1988), pp. 16311633 (in Russian).

[53] W. Weinelt, Untersuchungen zur Konvergenzgeschwindigkeit bei Differenzenverfahren, Zeitschrift der THK, 20 (1978), pp. 763-769.

[54] S. B. Zaitseva and A. A. Zlotnik, Sharp error estimates of vector splitting methods for the heat equation, Zh. Vychisl. Mat. Mat. Fiz., 39 (1999), pp. 472-491 (in Russian).

[55] A.A. Zlotnik, Convergence rate estimates in $L_{2}$ for projection-difference schemes approximating parabolic equations, Zh. Vychisl. Mat. Mat. Fiz., 18 (1978), pp. 1454-1465 (in Russian).

[56] A. A. Zlotnik, Convergence rate estimates for projection-difference schemes approximating second order hyperbolic equations, Vychisl. Prots. Sist., 8 (1991), pp. 116-167 (in Russian).

[57] A. A. Zlotnik, Some finite-element and finite-difference methods for solving mathematical physics problems with non-smooth data in an n-dimensional cube, Part I, Sov. J. Numer. Anal. Math. Modelling, 6 (1991), No. 5, pp. 421-451.

[58] A. A. Zlotnik, On superconvergence of a gradient for finite element methods for an elliptic equation with the nonsmooth right-hand side, Comput. Methods Appl. Math., 2 (2002), pp. 295-321. 